Recepción: 09 / 01 / 2017

Aceptación: 12 / 04 / 2017

Publicación: 15 / 07 / 2017

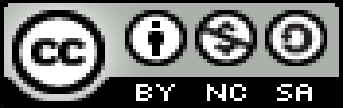

Ciencias económicas y empresariales

Artículo de investigación

\title{
La administración de empresas y la gestión de información en un acercamiento teórico
}

\section{Business management and information management in a theoretical approach}

\section{Gerenciamento de negócios e gerenciamento de informações em uma abordagem teórica}

\author{
Fabián E. Barcia-Villamar ${ }^{\mathrm{I}}$ \\ fabian_barcia@hotmail.com \\ Audrey J. Holguín-Briones II \\ audreyjac@hotmail.com \\ Homero M. Ferrin-Schettini ${ }^{\text {III }}$ \\ homeromanuelferrin@hotmail.com
}

Correspondencia: fabian_barcia@hotmail.com

\footnotetext{
I Magister en Contabilidad y Auditoría, Ingeniero en Contabilidad Pública y Auditoría, Contador Público, Docente Titular en la Universidad Laica Eloy Alfaro de Manabí, Manta, Ecuador.

II Magister en Contabilidad y Auditoría, Ingeniera en Contabilidad Pública y Auditoria, Docente Titular Auxiliar en la Universidad Laica Eloy Alfaro de Manabí, Manta, Ecuador.

III Magister en Negocios Internacionales y Gestión de Comercio Exterior, Diploma Superior en Gestión Empresarial Internacional, Diploma Superior en Economía del Ecuador y del Mundo, Diploma Superior en Economía Internacional, Ingeniero Civil, Arquitecto, Docente en la Universidad Laica Eloy Alfaro de Manabí, Manta, Ecuador.
} 


\title{
Resumen
}

En la administración de empresas, juega un papel primordial la información, ya que ésta se ha convertido en un recurso estratégico de preciado valor en nuestros días. La información se encuentra en todos los procesos de la empresa, permite la toma de decisiones, el estudio de la competencia, la comunicación con los públicos internos, entre otras cuestiones. Pero esa información es preciso organizarla, planificarla, dirigirla y controlarla. A partir de ello, en este trabajo se exponen algunos elementos de gestión de la información dentro de la administración en las empresas.

Palabras clave: administración de empresas; administración de la información; gestión empresarial; gestión de información.

\begin{abstract}
In business administration, information plays a key role, since it has become a strategic resource of precious value in our day and age. The information is found in all the processes of the company, allows decision making, the study of competition, communication with internal audiences, among other issues. But that information needs to be organized, planned, directed and controlled. From this, in this work some elements of information management within the administration in the companies are exposed.
\end{abstract}

Keywords: Business administration; information management; business management; information management.

\section{Resumo}

$\mathrm{Na}$ administração de empresas, a informação desempenha um papel fundamental, uma vez que se tornou um recurso estratégico de valor precioso em nosso dia e idade. A informação é encontrada em todos os processos da empresa, permite a tomada de decisões, o estudo da concorrência, a comunicação com o público interno, entre outras questões. Mas essa informação precisa ser organizada, planejada, dirigida e controlada. Deste modo, neste trabalho, alguns elementos de gerenciamento de informações dentro da administração nas empresas estão expostos.

Palavras chave: Administração de empresas; gerenciamento de informações; gestão empresarial; gerenciamento de informações. 


\section{Introducción}

En la administración de empresas juega un papel primordial la información, ya que ésta se ha convertido en un recurso estratégico de preciado valor en nuestros días.

"En el contexto de una empresa la gestión de información tiene como uno de sus aspectos más relevantes que permite la toma de decisiones" (Muñoz Cañavate A., 2003).

"La información ayuda a conocer la situación de la empresa y a analizar las estrategias que se ponen en marchar para su correcto funcionamiento. Las empresas necesitan estar al día y conocer datos relacionados con el funcionamiento y evolución del negocio, como el inventario, las ventas o la contabilidad de la organización. Sin embargo, también necesitan obtener otros datos relacionados con los consumidores, como sus hábitos de consumo, las necesidades y gustos de su mercado meta o qué tipo de estrategias son las más aceptadas por este colectivo" (EAE Business School, 2017).

Sánchez (2016), cita a Choo cuando plantea que una organización necesita aprender y solo aprende por la vía del procesamiento de información. Por tanto para la administración de empresas es una meta básica la gestión de información ya que permite potenciar sus recursos de información a fin de que la organización aprenda y se adapte a su ambiente cambiante.

Muchas empresas y organizaciones tienen éxitos en sus objetivos por la implantación de una gestión de información eficiente ya que es un ingrediente necesario para el éxito empresarial en el entorno global y dinámico de hoy, por lo tanto ésta debe constituir un campo de atención para la administración y gerencia de empresas. Esto significa que la administración apropiada de la información es un desafío importante para los gerentes. Así la gestión de la información representa:

- Un área funcional principal dentro de la empresa, que es tan importante para el éxito empresarial como las funciones de contabilidad, finanzas, administración de operaciones, marketing, y administración de recursos humanos.

- Una colaboración importante para la eficiencia operacional, la productividad, el servicio y la satisfacción del cliente. 
- Una fuente importante de respaldo para la toma de decisiones efectivas por parte de los gerentes.

- Un ingrediente importante para el desarrollo de productos y servicios competitivos que den a las organizaciones una ventaja estratégica en el mercado global.

A partir de ello, este trabajo tiene como objetivo exponer algunos elementos de gestión de la información en las empresas.

\section{Desarrollo}

Portieles (2011), cita a Gil (1997) cuando plantea "(...) Información es un dato o un conjunto de datos que, en un contexto determinado tienen un significado para alguien, y transmiten un mensaje útil en un lugar determinado. La información es un recurso primordial que incluso puede determinar el éxito o el fracaso de un negocio".

Por su parte, Hernández (2013) que cita a Ponjuán (2011), expone "la información es un elemento intangible pero cuantificable capaz de sostener la vida de las organizaciones en tanto constituya un recurso; es fuerza constitutiva de la sociedad que interactúa en forma permanente con otros elementos del ambiente; tiene efectos propios y regularidad; y gana en valor en la medida que es procesada, pudiendo ser objetivo de intercambio como mercancía, por tener valor de uso y valor de cambio, lo que la convierte en oferta de organizaciones especializada".

Rodríguez (2008) citado por Hernández (2013), señala con respecto al concepto de GI "Es también una actividad económica mediante la cual se trazan objetivos y estrategias, se organizan y distribuyen recursos, y se conducen y controlan acciones para un mejor manejo y uso de la información, de forma que se garantice eficaz y eficientemente, su identificación, obtención, representación, almacenamiento, búsqueda y recuperación, circulación o distribución, análisis y uso, con beneficios y la creación de ventajas competitivas para la organización".

A decir de Ponjuán (2014) la gestión de información tiene el objetivo de:

- Maximizar el valor y los beneficios derivados del uso de la información.

- Minimizar el costo de adquisición, procesamiento y uso de la información. 
- Determinar responsabilidades para el uso efectivo, eficiente y económico de la información.

- Asegurar un suministro continuo de la información.

La Gestión de Información, por tanto, es un proceso gerencial clave que posibilita la obtención, despliegue y utilización de recursos en una organización, con el objetivo de manejar eficaz y eficientemente la información y su optimización. Además posibilita el desarrollo y mantenimiento de las funciones propias de la gestión (planificación, organización, dirección y control), las cuales permiten tomar decisiones oportunas que ayuden al desarrollo de la entidad y logrando mayor eficacia y eficiencia en el logro de los objetivos, la misión y visión de la organización.

La Gestión de Información sin lugar a dudas tiene una marcada significación para las empresas e instituciones modernas, puesto que disponer de la información necesaria en el momento oportuno permitirá ofrecer mejores servicios a los usuarios, y además contribuirá al éxito y cumplimiento de los objetivos de la organización.

Artiles (2008) citado por Lugo (2013) refiere que, la gestión de información está asociada al sistema de información de una organización. En este sentido autores como Laudon, K y Laudon, J (2004) citados por Portieles (2011) definen los Sistemas de Información como "un conjunto de componentes interrelacionados que recolectan (o recuperan), procesan, almacenan y distribuyen información para apoyar la toma de decisiones y el control de una organización. Además de cumplir estas funciones los Sistemas de Información también pueden ayudar a los directivos y trabajadores a analizar problemas, a visualizar asuntos complejos y a crear productos nuevos".

A partir de los criterios analizados se puede definir el Sistema de Información como un conjunto de componentes que interactúan entre sí y funcionan como un todo. Este tiene entradas, procesos y salidas; donde también ocurre un proceso de retroalimentación. Se puede plantear que un Sistema de Información incluye como componentes principales: las personas, la información, los procesos y las tecnologías.

Los sistemas de información deben constituir una necesidad para las empresas, dado que el manejo de información relevante, actualizada y precisa, facilita el proceso de toma de decisiones y responde al cumplimiento de los objetivos y las metas trazados en cada entidad. 
Identificar los tipos de información que maneja la organización. Organizar los flujos de información a través de diagramas, los cuales constituyen los mapas de información en la empresa. Identificar el ciclo de vida de la información según los tipos de información. Identificar las necesidades de información por procesos de la organización. Identificar las necesidades de información personales según categorías (directivos, mandos medios, empleados). Diseñar y estructurar los servicios de información en función de las necesidades. Registrar, controlar y almacenar la información producida en la organización. Difundir y socializar la información para la generación de nuevos conocimientos.

La realización de una adecuada gestión de información en un Sistema de Información, favorecerá el trabajo con información para la toma de decisiones y la solución de problemas. La gestión de información ayuda al sistema a trabajar más eficientemente, ya que ambos se complementan, la gestión de información, se apoya en el sistema, y el sistema usa la gestión para dar respuesta a las necesidades informativas, brindar servicios de calidad, maximizar los beneficios y disminuir los costos.

Dentro de una empresa es fundamental determinar los flujos de información que no son más que los caminos y vías por las que transita la información de un punto a otro dentro de una organización. Estos pueden ser horizontales, verticales y transversales. Sus particularidades están dadas por los niveles de la organización (estratégico, táctico y operacional).

A decir de Rodríguez (2008), citado por Portieles (2011) la información constituye un recurso que puede localizarse, y a su vez, transmitirse fuera de la organización, por lo que pueden identificarse tres flujos informativos:

- Los flujos de información ambiental: conformados por la información proveniente del ambiente externo que ingresan en la organización por su importancia para el desarrollo del negocio.

- Los flujos de información interna: conformados por la información que, una vez que se convierte en un recurso organizacional, transita y se distribuye por la empresa, para aplicarse en los procesos que tienen lugar en ella. 
- Los flujos de información corporativa: conformados por la información que la organización envía hacia el ambiente externo, materializada en productos y servicios informativos.

Tener control de los flujos de información de una organización permite conocer aspectos relacionados con: la cantidad y el tipo de información que circula en el sistema, así como los diferentes subsistemas que lo integran; el papel de cada área funcional dentro de la organización, entre otros.

Según Cornella (2000), citado por Sánchez (2006) la eficiencia y calidad de un flujo "...depende que en el mismo se intercambie información más que solamente se transfiera (...) que quién emite y quién recibe se comuniquen, de forma que sintonicen sus contextos y sus intereses". La GI juega un papel primordial en el desarrollo de los flujos de información mediante el control y el análisis de los mismos, para la toma de decisiones y demás procesos de la organización.

A decir de Ponjuán (2014) "cuando intentamos potenciar el empleo de información en una organización debemos considerar que esta puede verse bajo diferentes enfoques. Estos enfoques tienen que ver con la esfera de acción de la información: no es igual la información que rodea a un individuo, que la que rodea a la organización internamente, ni la que se encuentra en el ambiente y le interesa. A esto le llamamos alcance de gestión de información". El alcance se materializa en los diferentes ambientes de gestión de información por los que están compuestas las organizaciones. Existen tres tipos de ambientes: ambiente de gestión de información individual, ambiente de gestión de información organizacional, y ambiente externo de información.

Siguiendo los elementos que conforman la GI se encuentra en la parte más operativa las herramientas que permiten el descubrimiento, monitoreo y evaluación de los recursos de información internos o externos con vistas a mejorar la GI y actuar de forma ventajosa ante la realidad. De estas operaciones se encarga la vigilancia, tal y como le explica Sánchez (2016) que cita a Salgado (2003) "se organiza como un sistema estructurado que permite coordinar las actividades de recuperación de la información, procesamiento análisis y diseminación, tanto de la información interna como del entorno, y todo, de acuerdo con un plan y una estrategia organizacional". 
Todas esas actividades de rastreo, captura, monitoreo, observación se consideran de vigilancia, pero al permitir la gestión, toma de decisiones y proyecciones futuras, así como adelantarse a cambios, se consideran inteligencia. Corroborando estos elementos Rodríguez(2007) considera que la Inteligencia se refiere a la capacidad que se posee para desarrollar procesos de tratamiento y uso de información y de conocimiento que faciliten una efectiva toma de decisiones, generando ventajas competitivas. En este conceptos válido ahondar en dos elementos y son los alusivos a proceso de tratamiento y uso de información, lo que apoya a la representación de la información, así como a los productos y servicios que de cada organización se genera.

Para una empresa, son primordiales las actividades de vigilancia e inteligencia empresarial. Así Palop y Vicente (1999), citado por Arencibia (2009), señalan que "antes de realizar cualquier actividad de vigilancia se deben acotar los aspectos básicos de un enfoque de vigilancia tecnológica y prospectiva, estos son: Objeto de la vigilancia; Qué se va a vigilar; Qué Informaciones buscar; Dónde localizarlas; Cómo se van a comunicar; A quién va dirigida y Qué medios se van a destinar".

Por su parte Eduardo Orozco (1998), citado por Sánchez (2006) define la Inteligencia Empresarial como "una herramienta gerencial cuya función es permitir a las administraciones el cumplimiento de la misión de sus organizaciones, mediante el análisis de la información relativa a su negocio y su entorno".

Se puede decir que la inteligencia empresarial consiste en la capacidad de examinar, estudiar, analizar e interpretar información, de manera que la organización pueda ser capaz de crear nuevos mercados, adelantarse a los cambios y tomar decisiones. Los procesos de vigilancia forman parte de la inteligencia empresarial. De manera concisa, el objetivo de la vigilancia es ofrecer información precisa a una persona o entidad en el momento adecuado sobre uno o un conjunto de temas determinados. Es por ello que hoy día, se utiliza con mucha frecuencia el término inteligencia en sustitución de vigilancia, pues la diferencia entre estos términos es imperceptible.

Ahora bien, dentro de esa gestión de información juega un papel primordial la auditoría de la información que consiste en un proceso que examina todas las funciones de la organización relacionadas con la información y determina como la utilizan. Constituye además un diagnóstico 
realizado a la organización de manera general, donde se ven reflejados aspectos referentes al uso de la información. Mediante ella se descubren, controlan y evalúan los flujos y recursos con que cuenta una organización.

El objetivo de las auditorias es asegurar que la información que circula por el sistema es la más apropiada para la organización, o sea se trata de que la organización reciba que la información relevante para sus intereses.

Efectivamente, las auditorías de información velan porque la información esté siendo bien tratada a partir de políticas de información que, aunque presenten particularidades en las organizaciones, velen por la confiabilidad de esa información que está siendo usada y certeza de que no está siendo falseada.

\section{Conclusiones}

La Gestión de Información es un proceso estratégico que en cualquier organización abarca todos los procesos y actividades de las empresas. La Gestión de Información forma parte de la administración de las empresas ya que sus componentes tienen una estrecha relación con el sistema en su conjunto.

Es por ello que los flujos de información, el ambiente en los que se desarrollan las organizaciones, la vigilancia e inteligencia y las auditorías de información, son elementos que competen a la Administración empresarial.

\section{Referencias bibliográficas}

Arencibia Fernández, Y. (2009). Observatorio de Mercados para las Tecnologías de la Información y las Comunicaciones. Unpublished Gestión de Información, Universidad de La Habana, La Habana.

EAE Business School. (2017). Tipos de sistemas de información empresarial. from http://www.eaeprogramas.es/empresa-familiar/tipos-de-sistemas-de-informacion-empresarial

Hernández Portal, E. (2013). Propuesta de mejoras para optimizar la Gestión de Información en el Centro de Información Bancaria y Económica (CIBE) del Banco Central de Cuba (BCC). Unpublished Gestión de Información, Universidad de La Habana, La Habana. 
Lugo, Y. (2013). Diseño del Cuadro de Mando de la Información para la Dirección de Producción de la Empresa de Gestión del Conocimiento y la Tecnología. (Tesis inédita de Licenciatura). Universidad de La Habana, La Habana.

Muñoz Cañavate A. (2003). Sistemas de información en las empresas. Hipertext.net, núm. 1.

Ponjuán Dante, G. (2011). La gestión de información y sus modelos representativos. Valoraciones. Ciencias de la Información. Instituto de Información Científica y Tecnológica La Habana, Cuba, 42(2), pp. 11-17.

Ponjuán, G. (2014). Introducción a la gestión de información. La Habana: Félix Varela.

Portieles Álvarez, A. A. (2011). Propuesta de perfeccionamiento del Sistema de Gestión de Información de la Agencia de Servicio Aduanales y Transitarios (ASAT). Unpublished Gestión de Información, Universidad de La Habana, La Habana

Rodríguez, Y. y Galán, E. (2007). La inteligencia organizacional: necesario enfoque de gestión de información y del conocimiento. Ciencias de la Información, 36(3). Recuperado de http://www.scielo.br/scielo.php?script=sci_arttext\&pid=S0100-19652007000300006

Sánchez Perdomo, D. (2006). Estado de la Gestión de la Información en empresas cubanas que han alcanzado alguna fase del Perfeccionamiento Empresarial. Unpublished Gestión de la información y el conocimiento, Universidad de La Habana, La Habana.

Sánchez M. (2016) Notas de clases Gestión de Información. Facultad de Comunicación. Universidad de la Habana. 